\title{
Bienestar psicológico en universitarios de México: prevención y atendimiento especializado
}

\author{
Psychological well-being in Mexican university students: prevention and \\ specialized care
}

Bem-estar psicológico em estudantes universitários mexicanos: prevenção e atendimento especializado

\section{Leticia Carreño Saucedo}

Profesora doctora de la Universidade Autónoma del Estado de México, Tejupilco, México

E-mail: psicoletty@hotmail.com ORCID: https://orcid.org/0000-0002-9221-6620

\section{Salvador Bobadilla Beltràn}

Profesor doctor de la Universidade Autónoma del Estado de México, Tejupilco, México

E-mail: sbb73@hotmail.com ORCID: https://orcid.org/0000-0003-1118-3431

Daniel Cardoso Jimenez

Profesor doctor de la Universidade Autónoma del Estado de México, Tejupilco, México

E-mail: dcj400_@hotmail.com ORCID: https://orcid.org/0000-0002-1762-1511

Recebido em 09 de agosto de 2021

Aprovado em 24 de setembro de 2021

Publicado em 27 de dezembro de 2021

\section{RESUMEN}

El bienestar psicológico en los universitarios tiene importancia para el desarrollo como estudiantes y como futuros profesionistas. Si se cuenta con un bienestar psicológico positivo se tendrán resultados favorables en todos los contextos donde se desenvuelvan. El objetivo de esta investigación fue analizar el bienestar psicológico en universitarios de la Normal de las licenciaturas de Educación especial y Prescolar, fue un estudio descriptivo y se utilizó un diseño no experimental, se aplicó la escala de Bienestar psicológico de Ryff con 6 dimensiones. Participaron en la investigación 151 universitarios de ambas licenciaturas, de 18 a 22 años, los cuales se encontraban cursando los semestres segundo, cuarto, sexto y octavo. Los resultados indican que en la dimensión auto aceptación en el nivel bajo con un $62 \%$ en y el $27 \%$ en un nivel alto. En crecimiento personal en el nivel medio con un $45 \%$, en un nivel alto con un $14 \%$. Es importante desarrollar programas de intervención que promuevan la asistencia educativa especializada para el bienestar psicológico de los universitarios. Además, la intervención en el nivel de prevención para bienestar de todos los integrantes de la institución, es urgente y necesario. Concluyendo, lo niveles medio y bajo fueron los que mayor puntaje obtuvieron en los universitarios, se tomar a consideración el contexto donde se encuentra ubicada la Normal Tejupilco, México con sus circunstancias que se enfrentan en el diario vivir.

Palabras clave: Bienestar psicológico, Servicio, Salud Mental, Prevención. 
http://dx.doi.org/10.5902/1984686X67125

\section{ABSTRACT}

The psychological well-being of university students is of importance for their development as students and future professionals. If it is based on positive psychological well-being, it will have favorable results in all contexts from which it develops. The objective of this research was to analyze the psychological well-being in college students of the Normal Special Education and Preschool Degrees, it was a descriptive study and an experimental design was used, applying the Ryff Psychological Well-being scale with 6 dimensions. 151 university students of both degrees, aged 18 to 22, who were in the second, fourth, sixth and eighth semesters, participated in the research. The results indicate that in the selfacceptance dimension at a low level with $62 \%$ and $27 \%$ at a high level. Personal growth at a medium level with $45 \%$, at a high level with $14 \%$. It is important to develop intervention programs that promote specialized educational assistance for the psychological well-being of university students. In addition, intervention at the level of prevention for the benefit of all members of the institution is urgent and necessary. In conclusion, the medium and low levels achieved the highest score obtained in university students, if the context in which it is located in Normal Tejupilco, Mexico is taken into account with the circumstances that they face in daily life.

Keywords: Psychological Wellness, Service, Mental Health, Prevention.

\section{RESUMO}

O bem-estar psicológico do estudante universitário é importante para o seu desenvolvimento como estudante e futuro profissional. Se for baseado no bem-estar psicológico positivo, terá resultados favoráveis em todos os contextos a partir dos quais se desenvolve. O objetivo desta pesquisa foi analisar o bem-estar psicológico em estudantes universitários dos cursos de Educação Normal Especial e Pré-Escolar, foi um estudo descritivo e utilizou-se um delineamento experimental, aplicando-se a escala de Bem-estar Psicológico de Ryff com 6 dimensões. Participaram da pesquisa 151 universitários de ambas as licenciaturas, com idades entre 18 e 22 anos. que estavam no segundo, quarto, sexto e oitavo semestres. Os resultados indicam que na dimensão autoaceitação está em um nível baixo com $62 \%$ e $27 \%$ em um nível alto. O crescimento pessoal a um nível médio com 45\%, a um nível alto com 14\%. É importante desenvolver programas de intervenção que promovam o atendimento educacional especializado para o bem-estar psicológico dos estudantes universitários. Além disso, a intervenção ao nível da prevenção em benefício de todos os membros da instituição é urgente e necessária. Em conclusão, os níveis médio e baixo alcançaram a pontuação mais alta obtida nos estudantes universitários, se o contexto em que está localizado em Normal Tejupilco, no México, for levado em consideração com as circunstâncias que enfrentam no dia a dia.

Palavras-chave: Bem-estar psicológico, Atendimento, Saúde Mental, Prevenção. 
http://dx.doi.org/10.5902/1984686X67125

\section{Introducción}

Desde la antigüedad, el hombre ha mostrado un gran interés por analizar el bienestar, se está convirtiendo en un asunto de interés científico en el ámbito de la psicología. Sólo a mediados del siglo pasado comenzaron a aparecer algunos estudios empíricos dentro de la salud y campos de conocimiento asociados (TAY; KUYKENDALL, 2013; VEENHOVEN, 1991). La mayoría de los estudios sobre la felicidad se encuentran en el campo de la psicología (GRAHAM, 2008) y la economía (ESTEBAN, 2013; KARLSSON; LYTTKENS; NILSSON, 2010;). Por ello, la Organización Mundial de la Salud (OMS) ha incluido y destacado la felicidad como un factor importante dentro del concepto de salud (CLONINGER; ZOHAR, 2011; FOWLER; CHRISTAKIS, 2008; OMS, 2004).

Preguntaron a las personas sobre los términos felicidad, utilidad, bienestar y satisfacción vital para ver si podían ser intercambiables y conocer el significado para ellas (ROBINSON; SHAVER; WRIGHTSMAN, 1991). Se realizó en el Survey Research Center (SRC) de la Universidad de Michigan y del National Opin-ion Research Center (NORC) de la Universidad de Chicago sobre, como se hizo en 1972 en la General Social Survey (GSS) de los Estados Unidos (EASTERLIN, 2003). La pregunta que se formuló fue: "En conjunto, ¿cómo diría que son las cosas en estos días: diría que es muy feliz, bastante feliz o no demasiado feliz?".

La felicidad es un objeto fundamental de la existencia humana (FOWLER; CHRISTAKIS, 2008), está determinada por un complejo conjunto de factores voluntarios e involuntarios (EASTERLIN, 2003). Aunque el término "felicidad" se emplea con frecuencia, suele restringirse a su significado contemporáneo puramente descriptivo, en el que se refiere simplemente a una condición psicológica (HAYBRON, 2016; LYUBOMIRSKY; KING; DIENER, 2005).

Los aristotélicos son menos entusiastas sobre la importancia de la "felicidad" en el sentido psicológico. Consideran obvio que la "felicidad" en el sentido de bienestar es lo único que importa para el bienestar humano (HAYBRON, 2016) y todo el comportamiento humano tiene como objetivo alcanzar la felicidad. El bienestar psicológico es un concepto que rescata la tradición iniciada por Aristóteles conocida como eudaimonismo.

Inicialmente, es necesario aclarar que la terminología utilizada para el tipo de valor en cuestión aquí, 'Bienestar', es diversa. 'Eudaimonia', 'Wel-fare' y 'flourishing' serán los términos utilizados lo que se refiere a cuestiones de beneficio y daño. 
http://dx.doi.org/10.5902/1984686X67125

El concepto de eudaimonía procede de la Ética a Nicómaco de Aristóteles (ARISTÓTELES, 2009), su obra filosófica sobre la "ciencia de la felicidad" (IRWIN, 2012). El nombre eudaimonismo deriva de la palabra griega 'eudaimonia', que a menudo se traduce como 'felicidad' (DECl; RYAN, 2008; HEINTZELMAN, 2018; HUTA; WATER-MAN, 2014), pero a veces se traduce como 'florecimiento'. De hecho, muchos estudiosos prefieren esta última traducción porque creen que capta mejor la preocupación de los antiguos griegos por la idea de vivir bien (DEIGH, 2010). Existen propuestas y teorías para entender el bienestar psicológico; entre ellas la propuesta por Jahoda (1958); Ryan y Deci (2001). Por lo tanto, no hay acuerdo para examinar el bienestar psicológico y falta de consenso entre los investigadores eudaimónicos sobre cómo deben ser sus medidas de bienestar (HAYBRON, 2016).

El bienestar psicológico forma parte de la salud y su implicación se revela en todas las esferas que establecen el ser humano: aspectos sociales, conocimientos y sentimientos, salud física y espiritualidad. El bienestar es un proceso de realización del daimon o verdadera naturaleza de (DECl; RYAN, 2008), es decir, de realización de los potenciales virtuosos y de vivir como se pretende intrínsecamente.

No existe una definición determinante del término, ya que este tema ha sido abordado desde diferentes enfoques, aunque se ha destacado el que propone Ryff (1989), que se considera uno de los más completos debido a su multidimensionalidad. El desarrollo teórico e investigativo que ha dominado la psicóloga a lo largo del tiempo, enfocado en las emociones negativas, y en la debilidad humana en general, proporcionando un molde que domina lo patógeno, aspecto que ha influido para que la psicología se identifique como psicopatología o psicoterapia (VÁZQUEZ et al., 2009).

Este énfasis en lo patológico fue influenciado por la Segunda Guerra Mundial, durante este tiempo, la psicología se fortaleció como un método dirigido a la restauración y reparación de daños (SELIGMAN, 2019), bajo el modelo biomédico dominante en ese momento.

Sin embargo, antes de la guerra, los objetivos de la psicología no sólo se orientaban a la curación de los trastornos mentales, sino que sus acciones debían contribuir a que los individuos fueran productivos y plenos, lo que implicaba desarrollar el talento y la inteligencia de las personas. Después de la guerra, los objetivos fueron más lentos y la psicología se centró en el tratamiento de las enfermedades mentales y el malestar humano (SELIGMAN; CSIKSZENTMIHALYI, 2014). 
http://dx.doi.org/10.5902/1984686X67125

\section{Psicología positiva}

En los últimos años, el cambio en la investigación en psicología, tiene un enfoque de atención para encontrar los aspectos positivos para trabajar preventivamente y dejar de lado los aspectos negativos y patológicos que son proverbialmente asimilados (SIMONTON; BAUMEISTER, 2005; VERA-VILLARROEL; GUERRERO, 2003).

Surge el concepto de psicología positiva (SELIGMAN, 1999). Maddux; Snyder y López (2004), mencionan que es un área que estudia sus características psicológicas activas y sus atributos positivos, las fortalezas y lo que está bien en los seres humanos (SELIGMAN, 1999). La psicología positiva defiende que la promoción de la salud mental implica el fomento de los recursos psicológicos, la mejora de la calidad de vida y la prevención de los trastornos mentales, especialmente aquellos trastornos que tienen una fuerte carga ambiental, promoviendo así la felicidad. Así, las aportaciones de la psicología positiva pueden y deben ser consideradas por los clínicos psiquiátricos y los de medicina general (ALMEDOM; GLANDON, 2007; DANNER; SNOWDON; FRIESEN, 2001; TAYLOR et al., 2000).

Para la psicología, los retos en el futuro próximo serán dedicarse a los aspectos positivos de la práctica humana, para concebir y vigorizar los componentes que permiten el progreso de los individuos, las comunidades y las sociedades, para mejorar la calidad de vida.

El bienestar se considera multidimensional, porque tiene en cuenta aspectos sociales y ambientales para la evaluación del bienestar (RYFF, 1989). Para este autor, el bienestar psicológico se diferencia en seis dimensiones

1) Valoración positiva de uno mismo;

2) Capacidad de gestionar eficazmente el entorno y la propia vida

3) Alta calidad de los vínculos personales

4) Afirmación de que la vida tiene intención y sentido;

5) Emoción que crece y se desarrolla a lo largo de la vida; $y$

6) Sentido de autodeterminación.

Las universidades y el bienestar psicológico, deben fomentar del bienestar, promoverse en todos los niveles educativos (BRIGHAM et al., 2018).

Los educadores tienen que enfrentarse a una serie de emociones cuando se relacionan con los alumnos (CHANG; DAVIS, 2009), y el apoyo continuo es esencial, incluyendo el desarrollo profesional (WEARE, 2006). La formación combinada de padres 
y profesores ha demostrado ser eficaz para la promoción de las competencias sociales en niños y adolescentes (WEBSTER; STRATTON et al., 2001).

El objetivo de la UNESCO, tal y como se recoge en la Estrategia de la UNESCO sobre Educación para la Salud y el Bienestar, es apoyar la contribución de los sectores educativos nacionales, promover una mejor salud y bienestar para todos los jóvenes. En particular, la Organización Mundial de la Salud subraya la importancia de implicar a todos los sectores a la hora de abordar la salud mental de los niños y adolescentes, ya que todos los sectores tienen un interés en el bienestar físico y mental presente y futuro de los jóvenes. (OMS, 2004).

Según la Organización Panamericana de la Salud y la Organización Mundial de la Salud (OMS, 2004), muchas universidades no aceptaron inmediatamente este reto de promover el bienestar de sus estudiantes. Sin embargo, el papel de la universidad es promover la salud con el objetivo de mejorar la vida de los involucrados, laboral y socialmente. Es importante promover etapas para la implantación de estilos de vida saludables en la comunidad universitaria (TSOUROS; DOWDING; DOORIS, 1998).

Para que se considere una universidad de calidad, se tienen en cuenta muchos aspectos que implican llegar a tiempo a las clases, realizar las tareas, participar en las reuniones de grupo, asistir a los seminarios, aprobar las pruebas de lectura o los exámenes.

La vida de los estudiantes universitarios con pruebas aplicadas por sus profesores, presiones del grupo al que pertenecen. (VÁZQUEZ et al., 2009). Además, al salir de casa, al moverse del lugar de origen para crear el futuro, la estudiante universitaria que está en los primeros ciclos puede perder el equilibrio emocional y ver afectado su bienestar personal y su estado de ánimo.

Es importante considerar que dentro de este grupo hay adolescentes, jóvenes y mujeres jóvenes que están en su desarrollo, y deben enfrentar situaciones rigurosas y responder positivamente a los requerimientos. (ESTEBAN; HERNÁNDEZ; QUISPE, 2017).

Se ha comprobado que en la adaptación y permanencia de los estudiantes universitarios al ámbito académico y los constituyentes que predominan en el ámbito académico son la autoestima, la inteligencia emocional, la dinámica familiar, el bienestar psicológico, dicha correspondencia es indiscutible y el problema va en aumento (ÁLVAREZ, 2007). 
http://dx.doi.org/10.5902/1984686X67125

\section{Métodos $^{1}$}

El objetivo de esta investigación fue analizar el bienestar psicológico en estudiantes universitarios de las carreras de educación especial y educación preescolar de la ciudad de Tejupilco, México.

La muestra estuvo conformada por 151 estudiantes de México, quienes llenaron esta encuesta en la fase preliminar de dos cursos en diferentes ambas licenciaturas. Esta población pertenece a diferentes comunidades de un entorno rural y semiurbano. Su edad estaba comprendida entre los 18 y los 22 años, con 83 participantes masculinos $(54,3 \%$ ) y 68 femeninos $(45,7 \%)$. Su edad media fue de 20 años. Se realizó una investigación descriptiva, con un diseño transversal no experimental. La participación fue voluntaria donde se les invito a participar explicando el fin de la investigación, además se les comento que su participación seria de manera anónima, y no se ofrecieron incentivos a los participantes.

\section{Medidas}

Se utilizó el instrumento Escala de bienestar psicológico que fue desarrollado por Ryff (1989). La versión original consta de seis dimensiones de 120 ítems cada una. En este estudio se utilizó la versión abreviada de Díaz et al (2006), compuesta por 39 ítems agrupados en las mismas seis dimensiones. La escala tiene un formato de respuesta tipo Likert de 6 opciones. (Auto aceptación, Relaciones positivas con los demás, Dominio del entorno, Propósito en la vida), siete ítems (Crecimiento personal) y ocho ítems (Autonomía). Se utilizó una escala de respuesta de seis puntos para todas las escalas, que van de 1 (totalmente en desacuerdo) a 6 (totalmente de acuerdo). Esta versión presenta propiedades psicométricas adecuadas como un alfa de Cronbach entre .70 y .83 para las 6 dimensiones.

\section{Resultados y discusión}

Se realizó separación por género, por licenciatura y después de la aplicación del instrumento de bienestar psicológico, se obtuvieron los siguientes resultados para cada una de las dimensiones; se tomaron en cuenta tres niveles de bienestar psicológico: bajo, medio $y$ alto.

La tabla 3 muestra las seis dimensiones del bienestar psicológico en sus tres niveles. 
Tabla 1 - Porcentaje por genero

\begin{tabular}{ccc}
\hline Genero & Participación & Porcentaje \\
\hline Femenino & 68 & $45.04 \%$ \\
Masculino & 83 & $54.96 \%$ \\
Total & 151 & $100 \%$ \\
\hline
\end{tabular}

Fuente: Elaboración propia (2020).

Tabla 2 - Distribución por licenciatura

\begin{tabular}{cccc}
\hline Licenciatura & Femenino & Masculino & Total \\
\hline Educación Especial & 29 & 49 & 78 \\
Educación Prescolar & 39 & 34 & 73 \\
Total & 68 & 83 & 151
\end{tabular}

Fuente: Elaboración propia (2020).

Tabla 3 - Resultados en porcentajes de las seis dimensiones del bienestar psicológico

\begin{tabular}{cccc}
\hline Dimensión & \multicolumn{3}{c}{ Nivel en porcentajes } \\
\hline Bujo aceptación & $62 \%$ & Medio & Alto \\
Relaciones positivas & $61 \%$ & $12 \%$ & $25 \%$ \\
Autonomía & $61 \%$ & $5 \%$ & $27 \%$ \\
Dominio del entorno & $82 \%$ & $7 \%$ & $34 \%$ \\
Crecimiento personal & $41 \%$ & $45 \%$ & $11 \%$ \\
Propósito de vida & $34 \%$ & $56 \%$ & $14 \%$ \\
\hline
\end{tabular}

Fuente: Elaboración propia (2020).

Esta investigación ha permitido identificar los niveles de bienestar psicológico en los estudiantes de la Normal de Tejupilco, México, obteniendo así el nivel a través de la Escala de Bienestar Psicológico de Ryff (Scales of Psychological Well-Being, SPWB), partiendo de los objetivos de conocer el nivel de bienestar psicológico entre los estudiantes 
http://dx.doi.org/10.5902/1984686X67125

universitarios. El 56\% de la población se encuentra en un nivel bajo, el $22 \%$ en un nivel medio y el 19\% tiene un bienestar psicológico alto.

Según la teoría planteada por algunos autores, existen factores que pueden contribuir al bajo o alto nivel de bienestar psicológico, entre ellos se encuentra la parte familiar que es una de las bases. En diferentes investigaciones se ha encontrado que el clima familiar positivo caracterizado por la cohesión afectiva entre padres e hijos, el apoyo, la confianza y la intimidad entre ellos y la comunicación familiar, motiva la conciliación conductual y psicológica de los estudiantes universitarios (GARCÍA; MUSITU; VEIGA, 2006, p.551).

Las causas del bajo bienestar psicológico en esta etapa de transición de la vida del estudiante están dadas por una compleja interacción entre factores objetivos y subjetivos. Los factores objetivos constituyen las condiciones externas: económicas, sociopolíticas, culturales, ambientales, que facilitan o dificultan su pleno desarrollo. Se abarca desde la ocupación, el estado civil, la escolaridad, la religión, que son factores que pueden influir en el bienestar psicológico de las personas. Los factores subjetivos están determinados por la evaluación que hacen de su propia vida y de esta etapa en particular.

En cuanto a los niveles de bienestar psicológico en la dimensión auto aceptación, se observa que el $62 \%$ de la población se encuentra en un nivel bajo.

Ryff (1989) menciona que las personas con baja auto aceptación no se sienten satisfechas con el yo, están en desacuerdo con lo que les sucedió en su pasado, se sienten inseguras de ciertas cualidades personales; y les gustaría ser diferentes a lo que son.

En los niveles de bienestar psicológico en la dimensión de Relaciones Positivas, el $61 \%$ de la población tiene un bajo bienestar psicológico, a lo que algunos aportes teóricos mencionan que quienes no tienen relaciones positivas con los demás "tienen pocas relaciones cercanas y de confianza; les resulta difícil abrirse y confiar en otras personas; están aislados y frustrados con respecto a sus relaciones personales" (RYFF, 1989, p. 1072).

Las condiciones económicas, políticas y sociales de la región del Sur del Estado de México, tiene limitaciones, a las cuales los estudiantes deben enfrentar. Tales como la vivienda y que representa un problema que el estudiante debe resolver al momento de ingresar a estudiar su licenciatura en el Municipio de Tejupilco, pues deben buscar un lugar para vivir, rentar, o buscar un familiar que les de alojamiento mientras se preparan por cuatro o cinco años para ser profesionistas. 
http://dx.doi.org/10.5902/1984686X67125

Otro aspecto a tener en cuenta es la dieta que cambia por completo ya que debido al estrés o a la falta de economía, a veces se saltan comidas o dejan de comer para ahorrar dinero.

Para entenderlo es importante contextualizar la ubicación de la escuela Normal. El municipio de Tejupilco Estado de México se ubica al suroeste, limita al norte con Otzoloapan, Zacazonapan, Temascaltepec, San Simón de Guerrero y Luvianos al sur con Amatepec y Sultepec, al este con San Simón de Guerrero, Texcaltitlán y Sultepec y al oeste con Michoacán, Guerrero y Luvianos. La Normal recibe estudiantes de los lugares mencionados, tomando en cuenta que muchos de ellos, es la primera vez que salen de casa por tanto tiempo, esto implica que traen consigo costumbres, hábitos, mitos que han sido heredados de sus generaciones.

Además, el descuido que muchas veces cometen errores, al estar influenciadas por malas compañías, que llevan al consumo de algunas sustancias como: alcohol, tabaco, drogas, desvelos, que suelen ser sus propios cómplices desde la universidad, esta mala decisión muchas veces dificulta su camino en su formación como profesional y por lo tanto su bienestar psicológico.

En las mujeres universitarias también surgen dificultades al salir de casa y encontrar un lugar para vivir solas, lo que hace que sientan la libertad y abusen de ella, provocando muchos embarazos a temprana edad. Todo ello provoca el abandono escolar y/o el bajo rendimiento académico.

El hecho de no estar constantemente pendientes de los tutores o de que los vean cada fin de semana les permite tener más libertad y desviar la atención de su objetivo que es convertirse en un profesional y así mismo, descuidar su bienestar psicológico.

De acuerdo a los datos obtenidos, se pudo identificar el nivel de bienestar psicológico de los estudiantes de la Escuela Normal de Tejupilco. Por lo tanto, se concluye que el nivel de bienestar psicológico en los estudiantes de la Normal de Tejupilco es bajo.

En los niveles medio y bajo de bienestar psicológico obtuvieron puntuaciones más altas. El crecimiento personal fue la dimensión predominante, en el nivel medio, seguido de la dimensión de propósito en la vida.

Los universitarios juegan un rol protagónico en la sociedad donde están inmersos. Si se orientan hacia el liderazgo y el desarrollo del conocimiento, son el escenario de la formación técnica y profesional de los miembros de la comunidad, a través de la educación, la re-investigación y la difusión del conocimiento, orientan y apoyan los cambios en diversos 
espacios nacionales e internacionales. Concluyendo el bienestar psicológico en los alumnos de la Normal de Tejupilco se encuentra en un nivel bajo.

Es importante que las políticas públicas universitarias trabajen a nivel de prevención en el bienestar de todos los miembros de la institución, porque de esta manera los estudiantes podrían ver desde el inicio de su formación profesional la importancia del bienestar psicológico en la vida cotidiana, para un buen desempeño en todas las áreas.

Estudios anteriores reportan que los estudiantes universitarios adultos de 30 a 55 años tienen un mayor nivel de auto aceptación, dominio del entorno, crecimiento personal y propósito en la vida, que los estudiantes jóvenes de 18 a 29 años; además, los nuevos estudiantes tendrían un mayor grado de auto aceptación y dominio del entorno (GARCíA, 2013).

Algunos autores como Librán (2006, p. 39) afirma que "el alto nivel de bienestar psicológico se forma al considerar los afectos positivos sobre los afectos negativos que provocan una experiencia inmediata".

En las dos últimas décadas se ha empezado a demostrar que los efectos psicológicos efectivos son una parte completa de la salud, pero pueden ser atribuidos a enfermedades y problemas físicos. Los observadores de la condición humana sostienen desde hace tiempo que los estados mentales positivos pueden conducir no sólo a una vida más plena y significativa, sino también a una existencia más saludable (TAYLOR et al., 2000, citado en VÁZQUEZ et al., 2009).

Existe mucha diversidad sobre el tema del bienestar psicológico, se considera un tema muy controvertido, algunas investigaciones al contrastar los resultados que se han obtenido, incluyen componentes cognitivos y sociales que investigan el crecimiento personal.

El bienestar psicológico es un estado percibido por el ser humano y se refiere al grado en que dicho individuo juzga su vida de manera general o global en términos positivos. Asimismo, lo que es el bienestar para un hombre o una mujer debe ser construido por ellos mismos, es una cuestión de experiencia propia e irrepetible, surgida en el marco de la vida social y la historia personal. 
http://dx.doi.org/10.5902/1984686X67125

\section{Conclusión}

Vinculándolos a los aspectos intrapersonales, así como contribuyendo a la relación causa-efecto cognitiva tanto para la felicidad existente en el intelecto como para la creatividad que surge de la ejecución de la tarea.

Los estudios parecen demostrar que el futuro de la creatividad en la intelectualidad pasa por trascender la visión sistemática y cognitiva, conduciendo así al pensamiento cuántico, alineando puntos aún no comprendidos, asociados a la conciencia y a la propia existencia. En este sentido, si queremos participar en la formación de jóvenes creativos que piensen y se comprometan críticamente con la ciencia y las soluciones inteligentes e innovadoras para el cambio sostenible, el sistema educativo debe cooperar asertivamente con sus universidades en un ambiente de respeto mutuo.

Según López; Herrero (2006), las universidades necesitan una formación profesional y un desarrollo cognitivo para desarrollar ciudadanos capaces de una creatividad colectiva con una perspectiva humanista para lograr un cambio paradigmático. Es necesario mejorar y sinergizar lo que el área ya posee -pruebas, evaluaciones, mapeos, modelos, métodos, etc. - con el entorno y la realidad humana. Los conocimientos adquiridos deben llegar al ámbito escolar, centrándose en la formación del profesorado y otras mejoras (SUÁREZ; WECHSLER, 2019).

Por ello, es urgente que la evidencia teórica aquí sintetizada sirva de guía para crear políticas públicas que redunden en una mayor inversión en educación. Se considera que tanto la inteligencia como la creatividad tienen una conexión epistemológica capaz de incidir directamente en los contextos educativos, dando lugar a una educación fundada en un nuevo paradigma de pensamiento que busca lo que está por venir y eleva la conciencia.

\section{Referencias}

ALMEDOM, A.; GLANDON, D. Resilience is not the absence of PTSD anymore than health is the absence of disease. Journal of Loss and Trauma, Massachusetts, v. 12, n. 2, p. 127-143, 2007. Disponible en:

https://www.tandfonline.com/doi/abs/10.1080/15325020600945962. Acceso en: 14 mayo 2021.

ÁLVAREZ, A. M. Textos Sociolingüísticos. Colección Textos Universitarios.1. ed. Venezuela: Publicaciones Vicerrectorado Académic, 2007. 
http://dx.doi.org/10.5902/1984686X67125

ARISTÓTELES. Ética à Nicômaco. 1. ed. Tradução do grego: Antonio de Castro Caeiro. São Paulo: Atlas, 2009.

BRIGHAM, T. et al. A Journey to Construct an All-Encompassing Conceptual Model of Factors Affecting Clinician Well-Being and Resilience. NAM Perspectives: expert voices in health \& Health Care, Washington, DC, v. 29, n. 2-8, 2018. DOI:

https://doi.org/10.31478/201801b. Disponible en: https://nam.edu/journey-constructencompassing-conceptual-model-factors-affecting-clinician-well-resilience/. Acceso en: 14 mayo 2021.

CHANG, M-L.; DAVIS, H. Understanding the Role of Teacher Appraisals in Shaping the Dynamics of Their Relationships with Students: Deconstructing Teachers' Judgments of Disruptive Behavior/Students. In: SCHUTZ, P. A.; ZEMBYLAS, M. (Eds.). Advances in Teacher Emotions Research: The Impact on Teachers Lives. New York: Springer, 2009, p. 95-127.

CLONINGER, C. R.; ZOHAR, A. Personality and the perception of health and happiness. Journal of Affect Disorders. v.128, n. 1-2, p. 24-32, 2011. Disponible en: https://www.sciencedirect.com/science/article/abs/pii/S0165032710004258. Acceso en: 17 mayo 2021.

DANNER, D. D., SNOWDON, D. A., FRIESEN, W. V. Positive emotions in early life and longevity: Findings from the nun study. Journal of Personality and Social Psychology, v. 80, n. 5, p.804-813, 2001. Disponible en: https://psycnet.apa.org/buy/2001-17232-009. Acceso en: 17 mayo 2021.

DECI, E. L.; RYAN, R. M. Hedonia, eudaimonia, and well-being: An introduction. Journal of Happiness Studies, v. 9, n. 1, p. 1-11, 2008. Disponible en:

https://link.springer.com/article/10.1007/s10902-006-9018-1. Acceso en: 17 mayo 2021.

DEIGH, J. Introducción a la ética Cambridge Introductions to Philosophy. Cambridge: Cambridge University Press, 2010.

DÍAZ, D. et al. Adaptación española de las escalas de bienestar psicológico de Ryff.

Psicothema, Oviedo, España, v. 18, n. 3, p. 572-577, 2006. Disponible en: https://www.redalyc.org/pdf/727/72718337.pdf Acceso en: 17 mayo 2021.

EASTERLIN, R. A. Explaining happiness. Procceedings of the National Academy of Sciences of the Unuted Stades of America. n.100, p. 11176-83, 2003.

ESTEBAN, R. F. C.; HERNÁNDEZ, R. M.; QUISPE, J. A. Bienestar psicológico y rendimiento académico en estudiantes de pregrado de psicología. Revista Internacional de In-vestigación En Ciencias Sociales. Asunción. v. 13, n. 2, p. 133-146, 2017. Disponible en https://dialnet.unirioja.es/servlet/articulo?codigo=6246945. Acceso en: 14 mayo 2021.

ESTEBAN, O-O. Happiness and Life Satisfaction. Our World In Data. 2013. Disponible en: https://ourworldindata.org/happiness-and-life-satisfaction. Acceso en: 17 mayo 2021. 
FOWLER, J. H.; CHRISTAKIS, N. A. Dynamic spread of happiness in a large social network: longitudinal analysis over 20 years in the Framingham Heart Study. BMJ, v. 337, p. 1-9, 2008. DOI: https://doi.org/10.1136/bmj.a2338. Disponible en: https://www.bmj.com/content/337/bmj.a2338. Acceso en: 17 mayo 2021.

GARCÍA, J. F.; MUSITU, G.; VEIGA, F. Autoconcepto en adultos de España y Portugal. Psicothema, Lisboa, v. 18, n. 3, p. 551-556, 2006. Disponible en: http://www.psicothema.com/psicothema.asp?id=3252. Acceso en: 17 mayo 2021.

GARCÍA, M. J. Competencias específicas en los estudios de derecho: definición y evaluación. Revista de Edu-cación y Derecho. Education and Law Review. Valladolid: Fundación Aranzadi Lex Nova, n. 11, 2013.

GRAHAM, C. Happiness and health: Lessons - And Questions - For Public Policy. Health Affaris (Millwood), v. 27, n. 1, p. 72-87, 2008. Disponible en: https://www.healthaffairs.org/doi/abs/10.1377/hlthaff.27.1.72 Acceso en: 17 mayo 2021.

HAYBRON, D. The Philosophical Basis of Eudaimonic Psychology. In: HAYBRON, D. M., Handbook of Eudaimonic Well-Being. International Handbooks of Quality-of-Life, Switzerland: Springer International Publishing, 2016.

HEINTZELMAN, S. J. Eudaimonia in the contemporary science of subjective well-being: Psychological well-being, self-determination, and meaning in life. In DIENER, E.; OISHI, S.; TAY, L. (editors), Handbook of well-being. Salt Lake City, UT: DEF Publishers, 2018.

HUTA, V.; WATERMAN, A. Eudaimonia and its distinction from hedonia: De-veloping a classification and terminology for understanding conceptual and opera-tional definitions. Journal of Happiness Studies, v. 15, n. 6, p. 1425-1456, 2014. Disponible en: https://link.springer.com/article/10.1007\%2Fs10902-013-9485-0 Acceso en: 17 mayo 2021.

IRWIN, T. H. Conceptions of happiness in the Nicomachean Ethics. In: SHIELDS, C. The Oxford Handbook of Aristotle. Oxford University Press, 2012.

JAHODA, M. Current concepts of positive mental health. New York, EE. UU: Basic Books, Inc., 1958.

KARLSSON, M.; LYTTKENS, C. H., NILSSON, T. Health. Happiness. Inequality. Expert Review Pharmacoeconomics and Outcomes Research. v.10, n. 5, p. 497-500, 2010. Disponible en: https://www.tandfonline.com/doi/abs/10.1586/erp.10.62. Acceso en: 17 mayo 2021.

LIBRÁN, E. C. Personality Dimensions and Subjective Well-Being. The Spanish journal of psychology. v. 9, n. 1, p. 38-44, 2006.Disponible en: https://www.cambridge.org/core/journals/spanish-journal-ofpsychology/article/abs/personality-dimensions-and-subjectivewellbeing/3372D4AB773A7617FCE0B43D01045B7F. Acceso en: 16 mayo 2021. 
LÓPEZ, J. S.; HERRERO, P. P. El nuevo modelo de formación continua en España: balance de un año de câmbios. Revista de Educación, n.341, p. 705-736, Septiembrediciembre 2006. Disponible en: https://sede.educacion.gob.es/publiventa/el-nuevomodelo-de-formacion-continua-en-espana-balance-de-un-ano-decambios/educacion/24264. Acceso en: 20 mayo 2021.

LYUBOMIRSKY, S.; KING, L.; DIENER, Ed. The Benefits of Frequent Positive Affect: Does Happiness Lead to Success? Psychological Bulletin, v. 131, n. 6, p. 803- 855, 2005. Disponible en: https://psycnet.apa.org/record/2005-15687-001?doi=1. Acceso en: 17 mayo 2021.

MADDUX, J.; SNYDER, C. R.; LOPEZ, S. Toward a positive clinical psycholo-gy: Deconstructing the illness ideology and constructing an ideology of human strengths and potential. In: LINLEY, P. A.; JOSEPH, S. (Eds). Positive psychology in practice Hoboken, NJ: Wiley, 2004, p. 320-334.

OMS. Organización Mundial de la Salud. Promoción de la Salud Mental. Conceptos, evidencia emergente, práctica. Organización Mundial de la Salud, Ginebra, 2004. Disponible en: https://www.who.int/mental_health/evidence/promocion_de_la_salud_mental.pdf Acceso en: 17 mayo 2021.

ROBINSON, J. P.; SHAVER, P. R.; WRIGHTSMAN, L. S. Measures of Personality and So-cial Psychological Attitudes. Measures of Social Psychological Atti-tudes. California: Academic Press, 1991.

RYAN, R. Y.; DECl, E. Sobre la felicidad y los potenciales humanos: una revisión de la investigación sobre el bienestar hedónico y eudaimónico. Psicología de las revisiones anuales, v. 52, p. 141-166, 2001.

RYAN, R.; DECl.; E. On happiness and human potentials: A review of research on hedonic and eudaimonic well-being. Annual Review of Psychology, v. 52, n.1, p. 141166, 2001. Disponible en:

https://www.annualreviews.org/doi/abs/10.1146/annurev.psych.52.1.141. Acceso en: 17 mayo 2021.

RYFF, C. D. In the eye of the beholder: Views of psychological well-being among middleaged and older adults. Psychology and Aging, v.4, n. 2, p. 195-210, 1989. Disponible en: http://doi.org/10.1037/0882-7974.4.2.195. Acceso en: 17 mayo 2021.

SELIGMAN, M. E. P. The president's address. American Psychologist, v. 54, n.8, p. 559-562, 1999.

SELIGMAN, M. E. P.; CSIKSZENTMIHALYI, M. Positive psychology: An introduction. Flow and the Foundations of Positive Psychology, p. 279-298, 2014. Disponible en: https://psycnet.apa.org/buy/2000-13324-001. Acceso en: 7 mayo 2021.

SIMONTON, D. K.; BAUMEISTER, R. Positive psychology at the Summit. Review of General Psychology. v. 9, n. 2, p. 99-102, 2005 Disponible en: https://journals.sagepub.com/doi/abs/10.1037/1089-2680.9.2.99. Acceso en: 7 mayo 2021. 
SUÁREZ, J.T.; WECHSLER, S. M. Identification of Creative and Intellectual Talent in the Classroom. Psicologia Escolar e Educacional, v. 23, 2019. Disponible en: https://www.scielo.br/j/pee/a/3Hc8K4y3D8ctxs5JsgzyjVQ/abstract/?lang=en. Acceso en: 17 mayo 2021.

TAY, L.; KUYKENDALL, L. Promoting happiness: the malleability of individual and societal subjective wellbeing. International Journal of Psychology, v. 48, n. 3, p. 159-176, 2013. Disponible en: https://www.tandfonline.com/doi/abs/10.1080/00207594.2013.779379. Acceso en: 7 mayo 2021.

TAYLOR, S. et al. Psychological resources, positive illusions, and health. American Psychologist, v. 55, n. 1, p. 99-109, 2000. DOI: http:// doi: 10.1037//0003-066x.55.1.99 Disponible en: https://psycnet.apa.org/fulltext/2000-13324-010.html . Acceso en: 7 mayo 2021.

TSOUROS, A. et al. Health Promoting Universities: concept, experience and framework for action. World health Organization Regional Office for Europe Copenhagen, 1998, 143 p. Disponible en: https://apps.who.int/iris/handle/10665/108095. Acceso: 7 mayo 2021.

VÁZQUEZ, C. et al. Bienestar psicológico y salud: aportaciones desde la psicología positiva. Anuario de Psicología Clínica y de La Salud, v. 5, n. 1, p. 15-28, 2009. Disponible en: http://dialnet.unirioja.es/servlet/articulo?codigo=3328166. Acceso en: 7 mayo 2021.

VEENHOVEN, R. Questions on happiness: classical topics, modern answers, blind spots. In: STRACK, F., ARGYLE, M., SCHWARZ, N. (editors). Subjective wellbeing, an interdisciplinary perspective. London: Pergamon Press, 1991.

VERA-VILLARROEL, P.; GUERRERO, A. Diferencias en habilidades de resolución de problemas sociales en sujetos optimistas y pesimistas. Universitas psychologica, Bogotá (Colombia), v. 2, n. 1, p. 21-26, enero-junio, 2003, p. 21-26.

WEARE, K. Taking a positive, holistic approach to the mental and emotional health and well-being of children and young people. In: NEWNES, C.; RADCLIFFE, N. (eds.) Making and Breaking Children's Lives. Ross-on-Wye, UK.: PCCS Books, 2006, p. 115-122.

WEBSTER-STRATTON, C.; REID, J.; HAMMOND, M. Preventing conduct problems, promoting social competence: a parent and teacher training partnership in head start. Journal of clinical child psychology. v. 30, n. 3, p. 283-302, 2001. DOI:

https://doi.org/10.1207/S15374424JCCP3003_2. Disponible en:

https://www.tandfonline.com/doi/abs/10.1207/S15374424JCCP3003_2. Acceso en: 13 junio 2021.

\section{Reconocimiento}

Esta investigación postdoctoral realizada por el primer y segundo autor, se llevó a cabo bajo la supervisión de profesora Carla L. Blum Vestena del Programa de Posgrado en Educación de la Universidade Estadual do Centro-Oeste (UNICENTRO), Brasil. Dicha 
investigación también contó con la colaboración del director de la Unidad Académica de Profesional Tejupilco de la UAEM.

\section{Notas}

${ }^{1}$ Los autores declaran no tener ningún conflicto de intereses en relación con la publicación de este trabajo.

cc) (i) (8) International (CC BY-NC 4.0) 